\title{
The measurements of the cosmic ray energy spectrum and the depth of maximum shower development of Telescope Array Hybrid trigger events
}

\author{
Heungsu Shin $^{a, *}$ on behalf of the Telescope Array Collaboration ${ }^{\dagger}$ \\ ${ }^{a}$ The University of Tokyo, Graduate School of Science, \\ Kashiwa, Chiba, Japan \\ E-mail: natrium@icrr.u-tokyo.ac.jp
}

The Telescope Array experiment is an ultra-high energy cosmic ray observatory located in Millard County, Utah, USA. The observatory consists of 3 fluorescence detector (FD) stations and 507 surface detectors (SD) that cover an area of $700 \mathrm{~km}^{2}$. Hybrid trigger is an external trigger system for the SD arrays that prompts the SD to perform data acquisition when an FD detects a shower-like event. In comparison with the SD autonomous trigger, hybrid trigger allows the SD to collect the data of an air shower that has primary energy below $10^{18.5} \mathrm{eV}$, where the efficiency of SD autonomous trigger decreases rapidly. We present the measurements of the cosmic ray energy spectrum and the depth of maximum shower development of hybrid trigger events observed from October 2010 to September 2014.

$37^{\text {th }}$ International Cosmic Ray Conference (ICRC 2021)

July 12th - 23rd, 2021

Online - Berlin, Germany

\footnotetext{
${ }^{*}$ Presenter

${ }^{\dagger}$ A complete list of collaborators can be found at the end of the proceedings
} 


\section{Introduction}

The Telescope Array (TA) experiment is the largest ultra high energy cosmic ray (UHECR) observatory placed in Millard County, Utah, USA that aims to investigate the origin and properties of UHECR[1]. The observatory consists of three fluorescence detector (FD) stations[2] that are surrounding the surface detector (SD) arrays that cover an area of $700 \mathrm{~km}^{2}$ [3]. A satellite photograph of the observatory indicating the positions of each detector and the observatory's location in USA are shown in the Figure 1.

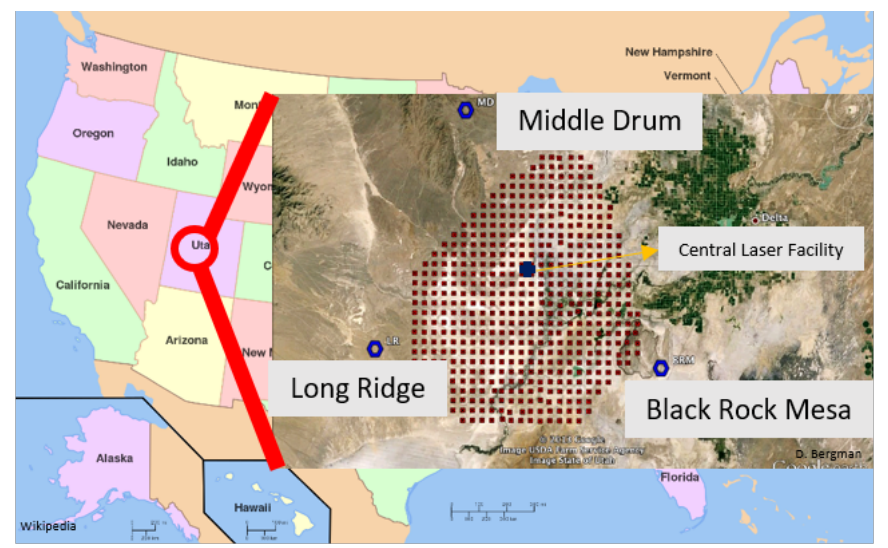

Figure 1: A satellite photograph of the Telescope Array observatory on a map of USA. The blue circles indicate the position of each FD station. From north clockwise, Middle Drum (MD) station, Black Rock Mesa (BRM) station, Long Ridge (LR) station. The red squares indicate the position of each SD of all 507 of them. The blue cross in the middle of the SD array indicates the position of Central Laser Facility (CLF). credit - D. Bergman, Wikipedia

Recent researches suggest that the composition of the cosmic rays with the primary energy of $10^{18.5} \mathrm{eV}$ is dominated by lighter nuclei[4], while the composition of the cosmic rays becomes heavier as the primary energy decreases to $10^{17} \mathrm{eV}[5]$. The transition might be caused by the difference in their origins. In order to investigate this transition further, the precise measurements of the energy spectrum and the depth of maximum shower development, $X_{\max }$ is critical. To achieve such precise observation, the hybrid reconstruction technique with the timing information of an SD in proximity of the core of extensive air shower (EAS) is employed. By using the timing information from the SD, it is possible to improve the accuracy in determination of shower geometry[6]. The TA collaboration implemented an external trigger system called "Hybrid trigger" in 2010. The hybrid trigger is issued by an FD station for SD array when an FD detects a shower-like event. This allows an SD subarray to collect the timing information from an extensive air shower with the primary energy below $10^{18.5} \mathrm{eV}$, where the efficiency of SD autonomous trigger decreases rapidly[3].

The energy threshold of the hybrid trigger system implemented in the MD station is different than that of the system implemented in BRM station and LR station. This is mainly due to the different distance between an FD station and a corresponding SD sub-array. Therefore, in this analysis, only hybrid trigger events observed in BRM station and LR station are used. 


\section{Monte Carlo study}

To simulate the EAS events, and the detector response for the hybrid trigger system, the Telescope Array collaboration has developed a dedicated Monte Carlo (MC) simulation framework. This MC study is important to evaluate the performance of the hybrid trigger system. The conditions and parameters used in the generation of the MC simulation set are summarized in the Table 1.

\begin{tabular}{lc}
\hline \hline Hybrid trigger MC generation conditions & Parameters \\
\hline Chemical composition & Proton, Iron \\
Energy step & $\operatorname{logE}=0.1$ \\
Energy range & $10^{17.5} \mathrm{eV}$ to $10^{19.2} \mathrm{eV}$ \\
Zenith angle & 0 degree to 60 degree \\
EAS core position & Inner $25 \mathrm{~km}$ radius from CLF \\
Atmospheric model & GDAS[7] \\
Simulation period & October 2010 to September 2014 \\
Hadronic interaction model & QGSJET-II-03 \\
Missing energy estimation & QGSJET-II-03 Proton \\
\hline
\end{tabular}

Table 1: Conditions and parameters used in the generation of the hybrid trigger MC.

In order to perform the reconstruction of hybrid trigger events, one SD is selected as the "anchor SD" that provides the least $\chi^{2}$ in the geometric reconstruction of the shower. In this analysis, we set the threshold for an "anchor SD" to be 3 MIPs. This is to prevent the contamination in geometric reconstruction caused by the chance particles.

Reconstruction results are filtered with the following criteria to obtain the results with high precision. This procedure is called "Quality cut" and the criteria used in this procedure are summarized in the Table 2.

\begin{tabular}{lc}
\hline \hline Quality cut items & Conditions \\
\hline Number of PMT with signal & $>20$ \\
Length of track & $>15$ degree \\
Zenith angle & $<57$ degree \\
Minimum viewing angle & $>20$ degree \\
$X_{\max }$ Bracketing & $X_{\text {start }}<X_{\max }<X_{\text {end }}$ \\
Additional volume cut & $X_{\text {start }}<550 \mathrm{~g} / \mathrm{cm}^{2}, X_{\text {end }}>850 \mathrm{~g} / \mathrm{cm}^{2}$ \\
\hline
\end{tabular}

Table 2: Quality cut items and criteria used in the reconstruction of the hybrid trigger events. Minimum viewing angle means the angle between the reconstructed shower axis and the FD.

One of the Quality cut criteria, Additional volume cut rejects the events with too deep observation start points $\left(X_{\text {start }}\right)$ and the events with too shallow observation end points $\left(X_{\text {end }}\right)$. Since those events have too little observed longitudinal development to reconstruct them precisely.

By comparing this filtered reconstruction results with the MC thrown parameters, it is possible to 
estimate the reconstruction accuracy for the hybrid trigger events. The primary energy reconstruction accuracy, the depth of maximum shower development $\left(X_{\max }\right)$ reconstruction accuracy, and the arrival direction reconstruction accuracy are shown in the Figure 2.

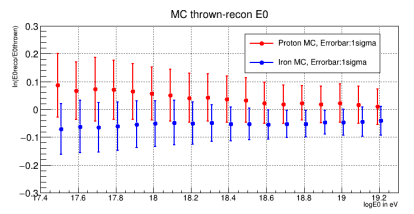

(a) E0 bias and resolution

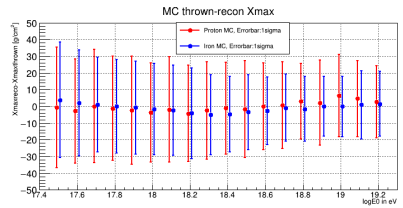

(b) $X_{\max }$ bias and resolution

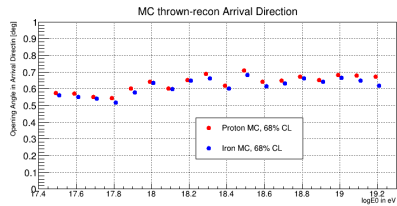

(c) Arrival direction accuracy

Figure 2: (a) The hybrid trigger reconstruction bias and resolution in primary energy E0 calculated by $\ln \left(\mathrm{E}_{\text {rec }} / \mathrm{E}_{\text {sim }}\right)$. Each distribution is fitted by a Gaussian distribution and $\sigma$ of the Gaussian distribution is indicated as the error bars. (b) The hybrid trigger reconstruction bias and resolution in $\mathrm{X}_{\max }$ calculated by $\mathrm{X}_{\max r e c}-\mathbf{X}_{\max \operatorname{sim}}$ Each distribution is fitted by a Gaussian distribution and $\sigma$ of the Gaussian distribution is indicated as the error bars. (c) The hybrid trigger reconstruction accuracy in Arrival direction indicated by the data points in opening angle distributions that are placed at the $68 \%$ of the distributions from 0 degree. All data points in the three sub-figures are shifted by -0.05 for proton and +0.05 for iron from the actual primary energies for visibility.

The accuracy of the hybrid trigger reconstruction in arrival direction is better than 0.8 degree with $68 \%$ of C.L. in the all simulated energy bins for both proton and iron.

The reconstruction biases in primary energy E0 differ with the composition and primary energy. For the lower primary energy of $10^{17.5} \mathrm{eV}$, the bias is around $\ln \left(\mathrm{E} 0_{\text {rec }} / \mathrm{E} 0_{\text {sim }}\right)=0.08$ for Proton, and $\ln \left(\mathrm{E} 0_{\text {rec }} / \mathrm{E} 0_{\text {sim }}\right)=-0.07$ for Iron. The reconstruction bias in primary energy decreases as primary energy increases. For the higher energy of $10^{19.2} \mathrm{eV}$, the bias is around $\ln \left(\mathrm{E} 0_{\text {rec }} / \mathrm{E} 0_{\text {sim }}\right)=0.02$ for proton, and $\ln \left(\mathrm{E}_{\text {rec }} / \mathrm{E}_{\text {sim }}\right)=-0.04$ for iron. The positive bias means the primary energy is overestimated in reconstruction and the negative bias means the primary energy is underestimated in reconstruction.

The reconstruction biases in $X_{\max }$ are within $-5 \mathrm{~g} / \mathrm{cm}^{2}$ to $7 \mathrm{~g} / \mathrm{cm}^{2}$ for all primary energy bins and compositions. The resolutions have the small energy dependence of $35 \mathrm{~g} / \mathrm{cm}^{2}$ at the primary energy of $10^{17.5} \mathrm{eV}$ and $20 \mathrm{~g} / \mathrm{cm}^{2}$ at the primary energy of $10^{19.2} \mathrm{eV}$.

\section{Results}

The hybrid trigger mode of FD operation started from October 8th 2010. Since the nature of fluorescence detection method, it is important to choose the data acquired in only clear nights. In this analysis, the method of visual confirmation of the cloud in the sky, called "WEAT code" is employed. From October 2010 to June 2019, BRM station recorded 3,297 hours and LR station recorded 2,900 hours of analyzable live time that makes total 6,197 hours of hybrid trigger observation.

For the first 4 years of observation, the total number of the hybrid trigger events after applying the quality cuts shown in the Table 2 and the aforementioned WEAT code is 2,774 events and 2,769 events for the BRM station and the LR station respectively that makes total 5,543 events. The 
energy distribution of the 5,543 hybrid trigger events is shown in the Figure 3-(a).

The hybrid trigger observation's BRM+LR total exposure for the proton and iron primary is calculated from the aperture, and the integrated observation time. The aperture of hybrid trigger for a certain energy bin $\mathrm{A} \Omega(E)$ can be written as,

$$
\mathrm{A} \Omega(E)=\frac{N_{r e c}(E)}{N_{\text {sim }}(E)} \times \mathrm{A}_{0} \Omega_{0}
$$

where $N_{\text {rec }}(E)$ is the number of reconstructed MC events in the energy bin after quality cut, $N_{\text {sim }}(E)$ is the number of simulated MC events in the energy bin. $\mathrm{A}_{0}$ represents the effective area that is determined by the maximum impact parameter, and $\Omega_{0}$ represents the solid angle that is determined by the maximum zenith angle. Therefore the total exposure for a certain energy bin is $\mathrm{A} \Omega(E) \mathrm{T}$, where $\mathrm{T}$ is the detector live time.

The first 4 years of hybrid trigger observation's BRM+LR total exposure for proton and iron primary is calculated and shown in the Figure 3-(b).
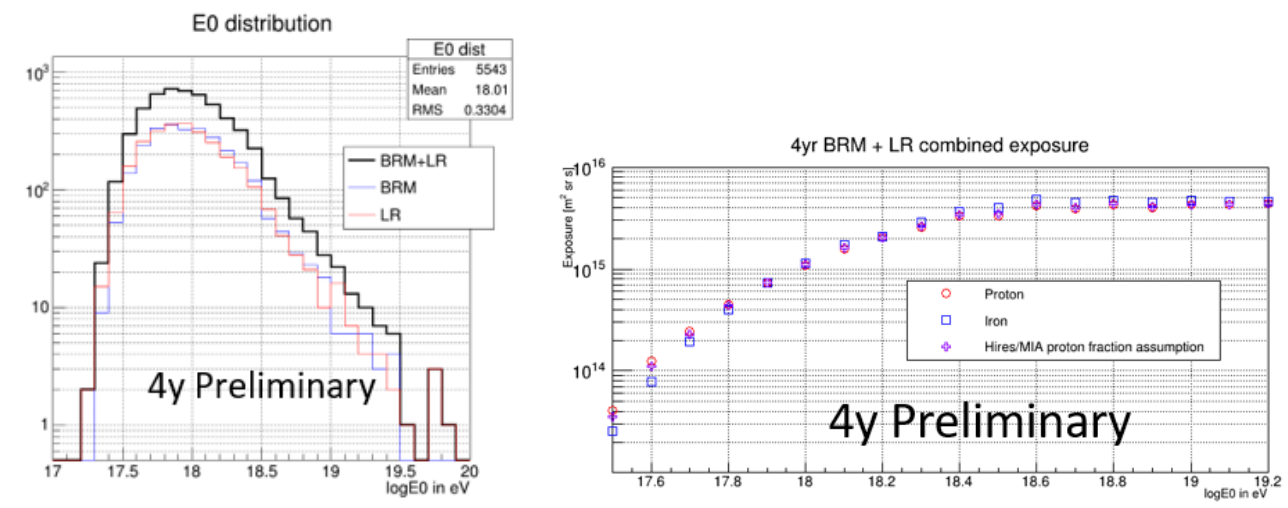

(a) E0 distribution for reconstructed (b) Combined exposure for proton and iron in 4 years observation. events in 4 years observation.

Figure 3: (a) The distribution of the number of reconstructed events for the first 4 years of hybrid trigger operation. Quality cuts and weather cuts are applied. The blue line indicates the events from BRM station, the red line indicates the events from LR station, the black line indicates the sum of both stations. (b) The combined exposure of BRM and LR stations for the first 4 years of observation as a function of energy. The red circles indicate the exposure for proton primary, and the blue squares indicate the exposure for iron primary. The purple crosses indicate the combined exposure where the HiRes and HiRes/MIA composition[8] is considered.

The spectrum of hybrid trigger observation is estimated by calculating the fluxes of cosmic rays for each energy bin. This can be written as,

$$
J(E)=\frac{N(E)}{A \Omega(E) T \Delta E}
$$

where $N(E)$ is the number of observed cosmic rays, and $\Delta E$ is the size of energy bin. The calculated spectrum of hybrid trigger observation for the first 4 years with the pure proton assumption is shown in the Figure 4-(a). In order to see the fine structure of the spectrum better, $E^{3} J(E)$ flux is also shown with the spectrum measurement result by FD monocular observation in the Figure 4-(b). 


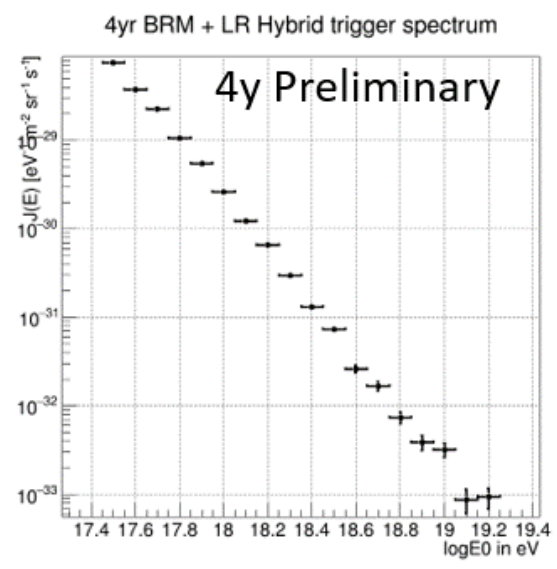

(a) Hybrid trigger spectrum $\mathrm{J}(\mathrm{E})$ in 4 years observation

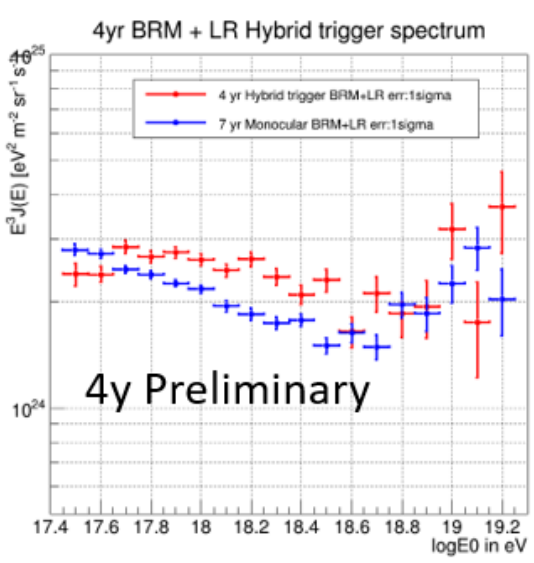

(b) Hybrid trigger spectrum $\mathrm{E}^{3} \mathrm{~J}(\mathrm{E})$ in 4 years observation with the spectrum measured by TA monocular in 7 years observation[9].

Figure 4: (a) The combined 4 years $J(E)$ spectrum measured by the hybrid trigger. HiRes and HiRes/MIA composition was assumed. Error bars indicate the $\sqrt{N}$ statistical error. (b) The combined 4 years $E^{3} J(E)$ spectrum measured by the hybrid trigger with the combined 7 years $E^{3} \mathrm{~J}(\mathrm{E})$ spectrum measured by the TA monocular. Hybrid trigger spectrum measurement in red dots. TA monocular spectrum measurement in blue dots. Error bars indicate indicate the $\sqrt{\mathrm{N}}$ statistical error. The same HiRes and HiRes/MIA composition was assumed for both.

The depth of maximum shower development, $X_{\max }$ is an important observable for studying the composition of UHECR. The distributions of reconstructed $X_{\max }$ of the proton and iron MC sets generated in the Section 2 were compared with the distribution of reconstructed $X_{\max }$ of the 4 years of observation data. Elongation rate of the observation data set and reconstructed MC set are also compared and shown in the Figure 5. As can be seen from the distributions shown in the Figure 5-(a), it is shown that the $X_{\max }$ distribution of 4 years observation data set is placed in between that of proton MC distribution and iron MC distribution. The elongation rate shown in the Figure 5-(b) indicates the existence of composition shift in the energy range from $10^{17.5} \mathrm{eV}$ to $10^{19.2} \mathrm{eV}$.

\section{Conclusion}

The TA hybrid trigger has been operating since October 2010 and recorded total 6,200 hours of observation with the BRM station and the LR station. The MC study shows that the primary energy resolutions $\ln \left(\mathrm{E}_{\text {rec }} / \mathrm{E}_{\text {sim }}\right)$ are estimated around 0.1 to 0.2 in the energy range from $10^{17.5} \mathrm{eV}$ to $10^{19.2} \mathrm{eV}$. The $X_{\max }$ resolutions are estimated around $20 \mathrm{~g} / \mathrm{cm}^{2}$ to $35 \mathrm{~g} / \mathrm{cm}^{2}$ in the above energy range. The preliminary hybrid trigger aperture has been estimated as well as the preliminary hybrid trigger spectrum. The preliminary result of measurement of the depth of maximum shower development indicates that the observed $X_{\max }$ is between the $X_{\max }$ of Proton MC and the Iron MC at the lower energy region. Then the observed $X_{\max }$ result gradually shifts to the $X_{\max }$ of Proton $\mathrm{MC}$ as the primary energy increases. 

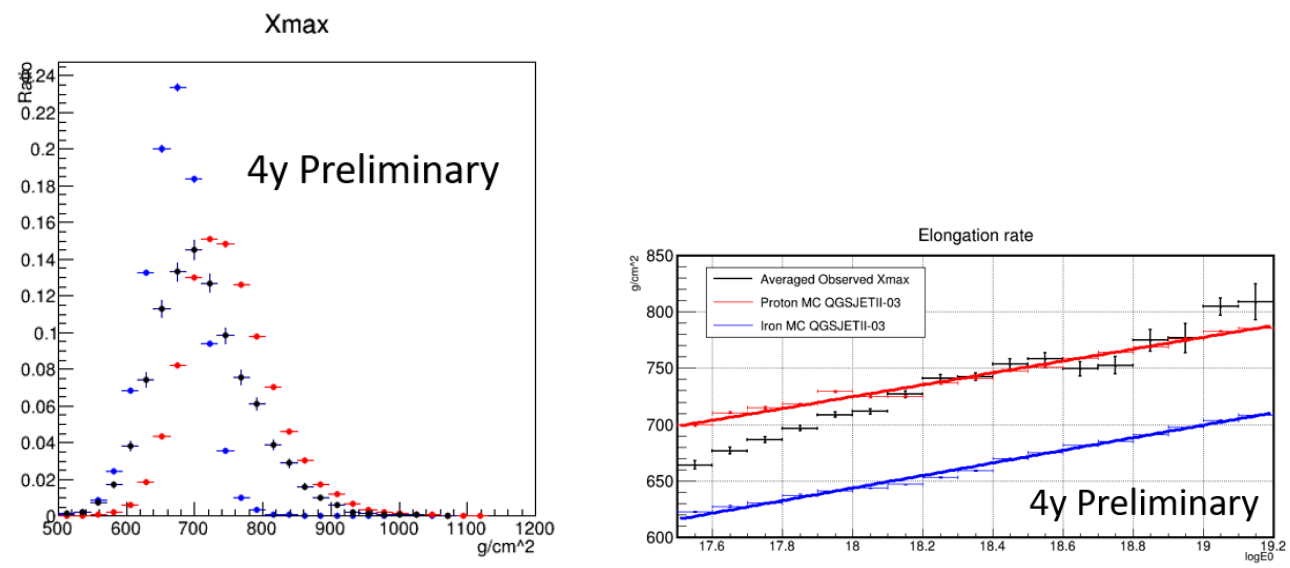

(a) $X_{\max }$ distributions of the reconstructed MC (b) Elongation rate plot of the reconstructed MC sets and the sets and the 4 years observation data set. $\quad 4$ years observation data set.

Figure 5: (a) The distributions of reconstructed $X_{\max }$ for proton MC, iron MC, and observation data. The red dots indicate the proton MC reconstruction. The blue dots indicate the iron MC reconstruction. The black dots indicate the observation data reconstruction. Error bars represent the statistical error. (b) The elongation rate plot of the same data. The red line indicates a linear fit for the proton MC reconstruction data points that are indicated as red dots. The blue line indicates a linear fit for the iron MC reconstruction data points that are indicated as blue dots. Error bars represent the statistical error.

\section{Acknowledgements}

The Telescope Array experiment is supported by the Japan Society for the Promotion of Science(JSPS) through Grants-in-Aid for Priority Area 431, for Specially Promoted Research JP21000002, for Scientific Research (S) JP19104006, for Specially Promoted Research JP15H05693, for Scientific Research (S) JP15H05741 and JP19H05607, for Science Research (A) JP18H03705, for Young Scientists (A) JPH26707011, and for Fostering Joint International Research (B) JP19KK0074, by the joint research program of the Institute for Cosmic Ray Research (ICRR), The University of Tokyo; by the Pioneering Program of RIKEN for the Evolution of Matter in the Universe (r-EMU); by the U.S. National Science Foundation awards PHY-1404495, PHY-1404502, PHY-1607727, PHY-1712517, PHY-1806797 and PHY-2012934; by the National Research Foundation of Korea (2017K1A4A3015188, 2020R1A2C1008230, \& 2020R1A2C2102800) ; by the Ministry of Science and Higher Education of the Russian Federation under the contract 075-15-2020-778, RFBR grant 20-02-00625a (INR), IISN project No. 4.4501.18, and Belgian Science Policy under IUAP VII/37 (ULB). This work was partially supported by the grants of The joint research program of the Institute for Space-Earth Environmental Research, Nagoya University and Inter-University Research Program of the Institute for Cosmic Ray Research of University of Tokyo. The foundations of Dr. Ezekiel R. and Edna Wattis Dumke, Willard L. Eccles, and George S. and Dolores Doré Eccles all helped with generous donations. The State of Utah supported the project through its Economic Development Board, and the University of Utah through the Office of the Vice President for Research. The experimental site became available through the cooperation of the Utah School and Institutional Trust Lands Administration (SITLA), U.S. Bureau of Land Management (BLM), 
and the U.S. Air Force. We appreciate the assistance of the State of Utah and Fillmore offices of the BLM in crafting the Plan of Development for the site. Patrick A. Shea assisted the collaboration with valuable advice and supported the collaboration's efforts. The people and the officials of Millard County, Utah have been a source of steadfast and warm support for our work which we greatly appreciate. We are indebted to the Millard County Road Department for their efforts to maintain and clear the roads which get us to our sites. We gratefully acknowledge the contribution from the technical staffs of our home institutions. An allocation of computer time from the Center for High Performance Computing at the University of Utah is gratefully acknowledged.

\section{References}

[1] H. Kawai et al., "Telescope array experiment," Nuclear Physics B - Proceedings Supplements, vol. 175-176, pp. 221-226, 2008. Proceedings of the XIV International Symposium on Very High Energy Cosmic Ray Interactions.

[2] H. Tokuno et al., "New air fluorescence detectors employed in the telescope array experiment," Nuclear Instruments and Methods in Physics Research Section A: Accelerators, Spectrometers, Detectors and Associated Equipment, vol. 676, pp. 54-65, 2012.

[3] T. Abu-Zayyad et al., "The surface detector array of the telescope array experiment," Nuclear Instruments and Methods in Physics Research Section A: Accelerators, Spectrometers, Detectors and Associated Equipment, vol. 689, pp. 87-97, 2012.

[4] R. Abbasi et al., "Study of ultra-high energy cosmic ray composition using telescope array's middle drum detector and surface array in hybrid mode," Astroparticle Physics, vol. 64, pp. 4962, 2015.

[5] T. Abu-Zayyad et al., "Measurement of the cosmic-ray energy spectrum and composition from 1017to 1018.3ev using a hybrid technique," The Astrophysical Journal, vol. 557, pp. 686-699, aug 2001.

[6] T. Abu-Zayyad et al., "Energy spectrum of ultra-high energy cosmic rays observed with the telescope array using a hybrid technique," Astroparticle Physics, vol. 61, pp. 93-101, 2015.

[7] ARL-NOAA, "Global data assimilation system (gdas1) archive information," 2004.

[8] R. Abbasi et al., "Studies of systematic uncertainties in the estimation of the monocular aperture of the hires experiment," Astroparticle Physics, vol. 27, no. 5, pp. 370-381, 2007.

[9] R. Abbasi et al., "The energy spectrum of cosmic rays above 1017.2 ev measured by the fluorescence detectors of the telescope array experiment in seven years," Astroparticle Physics, vol. 80, pp. 131-140, 2016. 


\section{Full Authors List: Telescope Array Collaboration}

R.U. Abbasi ${ }^{1}$, T. Abu-Zayyad ${ }^{1,2}$, M. Allen ${ }^{2}$, Y. Arai $^{3}$, R. Arimura ${ }^{3}$, E. Barcikowski ${ }^{2}$, J.W. Belz ${ }^{2}$, D.R. Bergman ${ }^{2}$, S.A. Blake ${ }^{2}$, I. Buckland ${ }^{2}$, R. $\mathrm{Cady}^{2}$, B.G. Cheon ${ }^{4}$, J. Chiba ${ }^{5}$, M. Chikawa ${ }^{6}$, T. Fujii ${ }^{7}$, K. Fujisue ${ }^{6}$, K. Fujita ${ }^{3}$, R. Fujiwara ${ }^{3}$, M. Fukushima ${ }^{6}$, R. Fukushima ${ }^{3}$, G. Furlich ${ }^{2}$, R. Gonzalez ${ }^{2}$, W. Hanlon ${ }^{2}$, M. Hayashi ${ }^{8}$, N. Hayashida ${ }^{9}$, K. Hibino ${ }^{9}$, R. Higuchi ${ }^{6}$, K. Honda ${ }^{10}$, D. Ikeda ${ }^{9}$, T. Inadomi ${ }^{11}$, N. Inoue ${ }^{12}$, T. Ishii ${ }^{10}$, H. Ito ${ }^{13}$, D. Ivanov ${ }^{2}$, H. Iwakura ${ }^{11}$, A. Iwasaki ${ }^{3}$, H.M. Jeong ${ }^{14}$, S. Jeong ${ }^{14}$, C.C.H. Jui ${ }^{2}$, K. Kadota ${ }^{15}$, F. Kakimoto ${ }^{9}$, O. Kalashev ${ }^{16}$, K. Kasahara ${ }^{17}$, S. Kasami ${ }^{18}$, H. Kawai ${ }^{19}$, S. Kawakami ${ }^{3}$, S. Kawana ${ }^{12}$, K. Kawata ${ }^{6}$, I. Kharuk ${ }^{16}$, E. Kido ${ }^{13}$, H.B. Kim ${ }^{4}$, J.H. Kim ${ }^{2}$, J.H. Kim ${ }^{2}$, M.H. Kim ${ }^{14}$, S.W. Kim ${ }^{14}$, Y. Kimura ${ }^{3}$, S. Kishigami ${ }^{3}$, Y. Kubota ${ }^{11}$, S. Kurisu ${ }^{11}$, V. Kuzmin ${ }^{16}$, M. Kuznetsov ${ }^{16,20}$, Y.J. Kwon ${ }^{21}$, K.H. Lee ${ }^{14}$, B. Lubsandorzhiev ${ }^{16}$, J.P. Lundquist ${ }^{2,22}$, K. Machida ${ }^{10}$, H. Matsumiya ${ }^{3}$, T. Matsuyama ${ }^{3}$, J.N. Matthews ${ }^{2}$, R. Mayta ${ }^{3}$, M. Minamino ${ }^{3}$, K. Mukai ${ }^{10}$, I. Myers ${ }^{2}$, S. Nagataki ${ }^{13}$, K. Nakai ${ }^{3}$, R. Nakamura ${ }^{11}$, T. Nakamura ${ }^{23}$, T. Nakamura ${ }^{11}$, Y. Nakamura ${ }^{11}$, A. Nakazawa ${ }^{11}$, E. Nishio ${ }^{18}$, T. Nonaka ${ }^{6}$, H. Oda ${ }^{3}$, S. Ogio $^{3,24}$, M. Ohnishi ${ }^{6}$, H. Ohoka ${ }^{6}$, Y. Oku ${ }^{18}$, T. Okuda ${ }^{25}$, Y. Omura ${ }^{3}$, M. Ono ${ }^{13}$, R. Onogi ${ }^{3}$, A. Oshima ${ }^{3}$, S. Ozawa ${ }^{26}$, I.H. Park ${ }^{14}$, M. Potts ${ }^{2}$, M.S. Pshirkov ${ }^{16,27}$, J. Remington ${ }^{2}$, D.C. Rodriguez ${ }^{2}$, G.I. Rubtsov ${ }^{16}$, D. Ryu ${ }^{28}$, H. Sagawa ${ }^{6}$, R. Sahara ${ }^{3}$, Y. Saito ${ }^{11}$, N. Sakaki ${ }^{6}$, T. Sako ${ }^{6}$, N. Sakurai ${ }^{3}$, K. Sano ${ }^{11}$, K. Sato ${ }^{3}$, T. Seki ${ }^{11}$, K. Sekino ${ }^{6}$, P.D. Shah ${ }^{2}$, Y. Shibasaki ${ }^{11}$, F. Shibata ${ }^{10}$, N. Shibata ${ }^{18}$, T. Shibata ${ }^{6}$, H. Shimodaira ${ }^{6}$, B.K. Shin ${ }^{28}$, H.S. Shin ${ }^{6}$, D. Shinto ${ }^{18}$, J.D. Smith ${ }^{2}$, P. Sokolsky ${ }^{2}$, N. Sone ${ }^{11}$, B.T. Stokes ${ }^{2}$, T.A. Stroman ${ }^{2}$, Y. Takagi ${ }^{3}$,

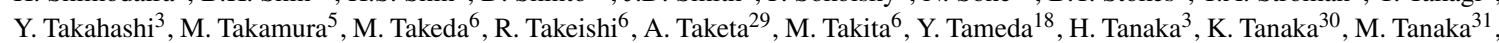
Y. Tanoue ${ }^{3}$, S.B. Thomas ${ }^{2}$, G.B. Thomson ${ }^{2}$, P. Tinyakov ${ }^{16,20}$, I. Tkachev ${ }^{16}$, H. Tokuno ${ }^{32}$, T. Tomida ${ }^{11}$, S. Troitsky ${ }^{16}$, R. Tsuda ${ }^{3}$, Y. Tsunesada ${ }^{3,24}$, Y. Uchihori ${ }^{33}$, S. Udo ${ }^{9}$, T. Uehama ${ }^{11}$, F. Urban ${ }^{34}$, T. Wong ${ }^{2}$, K. Yada $^{6}$, M. Yamamoto ${ }^{11}$, K. Yamazaki ${ }^{9}$, J. Yang ${ }^{35}$, K. Yashiro ${ }^{5}$, F. Yoshida ${ }^{18}$, Y. Yoshioka ${ }^{11}$, Y. Zhezher ${ }^{6,16}$, and Z. Zundel ${ }^{2}$

${ }^{1}$ Department of Physics, Loyola University Chicago, Chicago, Illinois, USA

${ }^{2}$ High Energy Astrophysics Institute and Department of Physics and Astronomy, University of Utah, Salt Lake City, Utah, USA

${ }^{3}$ Graduate School of Science, Osaka City University, Osaka, Osaka, Japan

${ }^{4}$ Department of Physics and The Research Institute of Natural Science, Hanyang University, Seongdong-gu, Seoul, Korea

${ }^{5}$ Department of Physics, Tokyo University of Science, Noda, Chiba, Japan

${ }^{6}$ Institute for Cosmic Ray Research, University of Tokyo, Kashiwa, Chiba, Japan

7 The Hakubi Center for Advanced Research and Graduate School of Science, Kyoto University, KitashirakawaOiwakecho, Sakyo-ku, Kyoto, Japan

${ }^{8}$ Information Engineering Graduate School of Science and Technology, Shinshu University, Nagano, Nagano, Japan

${ }^{9}$ Faculty of Engineering, Kanagawa University, Yokohama, Kanagawa, Japan

${ }^{10}$ Interdisciplinary Graduate School of Medicine and Engineering, University of Yamanashi, Kofu, Yamanashi, Japan

${ }^{11}$ Academic Assembly School of Science and Technology Institute of Engineering, Shinshu University, Nagano, Nagano, Japan

${ }^{12}$ The Graduate School of Science and Engineering, Saitama University, Saitama, Saitama, Japan

13 Astrophysical Big Bang Laboratory, RIKEN, Wako, Saitama, Japan

${ }^{14}$ Department of Physics, SungKyunKwan University, Jang-an-gu, Suwon, Korea

${ }^{15}$ Department of Physics, Tokyo City University, Setagaya-ku, Tokyo, Japan

${ }^{16}$ Institute for Nuclear Research of the Russian Academy of Sciences, Moscow, Russia

${ }^{17}$ Faculty of Systems Engineering and Science, Shibaura Institute of Technology, Minato-ku, Tokyo, Japan

${ }^{18}$ Department of Engineering Science, Faculty of Engineering, Osaka Electro-Communication University, Neyagawashi, Osaka, Japan

${ }^{19}$ Department of Physics, Chiba University, Chiba, Chiba, Japan

${ }^{20}$ Service de Physique Théorique, Université Libre de Bruxelles, Brussels, Belgium

${ }^{21}$ Department of Physics, Yonsei University, Seodaemun-gu, Seoul, Korea

22 Center for Astrophysics and Cosmology, University of Nova Gorica, Nova Gorica, Slovenia

${ }^{23}$ Faculty of Science, Kochi University, Kochi, Kochi, Japan

${ }^{24}$ Nambu Yoichiro Institute of Theoretical and Experimental Physics, Osaka City University, Osaka, Osaka, Japan

${ }^{25}$ Department of Physical Sciences, Ritsumeikan University, Kusatsu, Shiga, Japan

${ }^{26}$ Quantum ICT Advanced Development Center, National Institute for Information and Communications Technology, Koganei, Tokyo, Japan

27 Sternberg Astronomical Institute, Moscow M.V. Lomonosov State University, Moscow, Russia

${ }^{28}$ Department of Physics, School of Natural Sciences, Ulsan National Institute of Science and Technology, UNIST-gil, 
Ulsan, Korea

${ }^{29}$ Earthquake Research Institute, University of Tokyo, Bunkyo-ku, Tokyo, Japan

${ }^{30}$ Graduate School of Information Sciences, Hiroshima City University, Hiroshima, Hiroshima, Japan

${ }^{31}$ Institute of Particle and Nuclear Studies, KEK, Tsukuba, Ibaraki, Japan

${ }^{32}$ Graduate School of Science and Engineering, Tokyo Institute of Technology, Meguro, Tokyo, Japan

33 Department of Research Planning and Promotion, Quantum Medical Science Directorate, National Institutes for Quantum and Radiological Science and Technology, Chiba, Chiba, Japan

${ }^{34}$ CEICO, Institute of Physics, Czech Academy of Sciences, Prague, Czech Republic

${ }^{35}$ Department of Physics and Institute for the Early Universe, Ewha Womans University, Seodaaemun-gu, Seoul, Korea

* Deceased 